\title{
COVID-19 and COVID-like Patients: A Brief Analysis and Findings of Two Deceased Cases
}

Giosuè Mario Balzanelli ${ }^{1}$, Pietro Distratis ${ }^{1}$, Sergey K. Aityan ${ }^{2}$, Felice Amatulli ${ }^{1}$, Orazio Catucci ${ }^{1}$, Angelo Cefalo ${ }^{1}$, Gianna Dipalma ${ }^{3}$, Francesco Inchingolo ${ }^{3}$, Rita Lazzaro ${ }^{1}, K_{i e u}$ C. D. Nguyen ${ }^{4}$, Davide Palazzo ${ }^{1}$, Van Pham Hung ${ }^{5}$, Diego Tomassone ${ }^{6}$, Toai Tran Cong ${ }^{7}$, Ciro Gargiulo Isacco ${ }^{1,3,4}$

\begin{abstract}
${ }^{1}$ Department of Pre-hospital and Emergency, SG Giuseppe Moscati Hospital, Taranto City, Italy; ${ }^{2}$ Department of Interdisciplinary Medicine, "Aldo Moro" University of Bari, School of Medicine D.I.M, Bari City, Italy; ${ }^{3}$ Department of Multidisciplinary Research Centre, Lincoln University, Oakland, California, USA; ${ }^{4}$ American Stem Cells Hospital and Human Stem cells Institute, Ho Chi Minh City, Vietnam; ${ }^{5}$ Phan Chau Trinh University of Medicine and Nam-Khoa Biotek, Ho Chi Minh City, Vietnam; ${ }^{6}$ Nutritherapy Research Center, Urbino City, Italy; ${ }^{7}$ Department of Histology, Embryology and Genetics, Pham Ngoc Thach University of Medicine, Ho Chi Minh City, Vietnam
\end{abstract}

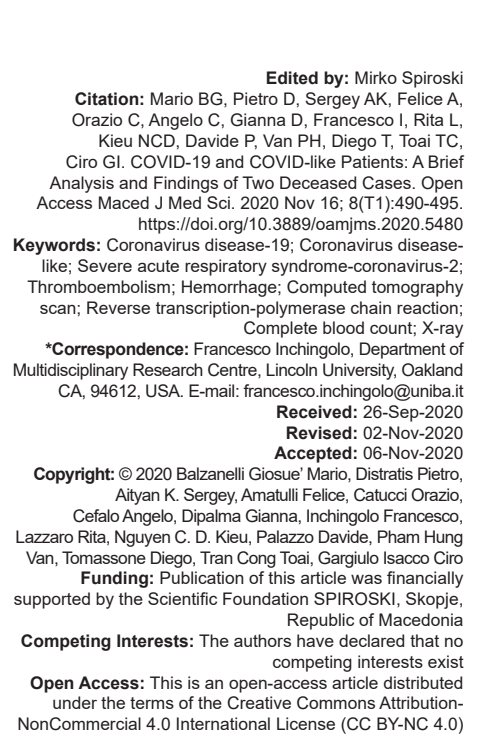

\begin{abstract}
BACKGROUND: The predominant pattern of lung lesions in patients affected by coronavirus disease (COVID19) disease is diffuse alveolar damage with massive thromboembolism similar as described in patients infected with severe acute respiratory syndrome coronavirus and Middle East respiratory syndrome coronaviruses. Hyaline membrane formation and pneumocyte atypical hyperplasia were frequent. Importantly, the formation of plateletfibrin thrombi in small vessels was seen consistent with coagulopathy, which appeared to be a common feature in patients who died of COVID-19. However, many were the cases found with similar COVID-19 symptomatology though negative results from nasal-pharyngeal swab performed by reverse transcription-polymerase chain reaction (RT-PCR). This latter typology of patients, otherwise named COVID-like, showed analogous clinical signs with similar arterial blood gas, cell blood count and laboratory parameters, and same computed tomography (CT)-scan groundglass opacities. Symptoms such as cough, fever, and difficulty breathing were highly similar as well. Both forms, COVID-19 and COVID-like, are primarily respiratory with multi-organ involvement and both revealed comparable incubation periods often with a rapid onset and unexpected decay.

CASE REPORT: In this brief paper, we described two cases regarding two deceased males, one confirmed COVID19 (RT-PCR but not CT scan) and the second a COVID-like (negative for RT-PCR but positive to CT scan with ground-glass opacity) whom condition, disease patterns, and analysis were highly similar.

CONCLUSION: Improved investigation is mandatory, in which RT-PCR and CT scan procedures are completed by data from more detailed laboratory analysis, ABG analysis, BALF, and a deeper clinical assessment.
\end{abstract}

\section{Introduction}

At the beginning of the current year, Italy was suddenly involved in the current coronavirus disease (COVID)-19 pandemics, and many thousands of patients have been recorded [1]. Nowadays, the main accepted procedure to confirm the positivity to COVID-19 is based on the nasopharyngeal swab analyzed by reverse transcription-polymerase chain reaction reverse transcription-polymerase chain reaction (RT-PCR). However, the limits of this procedure in diagnosing the disease are well known. The main issue is related to the low specificity of the procedure in verifying the presence of both false negatives and false positives; the second issue is due to mistakes related to the manual procedure by the health personnel; and third the possibility that virus has moved downward and settled within lungs, generating negative swab results. In addition, specimen tested without proper internal control could bias the results [2], [3], [4].

In our experience, the presence of COVIDlike patients represented an important number $(n \geq 60)$ (data not showed) and 2 patients out of 60 underwent bronchi-alveolar liquid test (bronchoalveolar lavage fluid [BALF]) resulting positive to severe acute respiratory syndrome coronavirus (SARS-CoV-2). Therefore, we do not exclude that among COVID-like patients may remain someone that was eventually infected by SARS-CoV-2 but resulted negative to the swab screen. Nevertheless, clinical and laboratory outcomes showed substantial similarities between SARS-CoV-2 and COVID-like patients which sharing pathophysiological features related to specific traits of severe and interstitial 
pneumonia with clinical presentation greatly resembling each other's [4], [5].

It follows that the RT-PCR negative results should not be conclusive of SARS-CoV-2 infection-free status and should not be used as solely indicator in the decision making treatment plan in suspected COVID-19 cases. Hence, we proposed a new diagnostic protocol that eventually includes the clinical observations, patient history, BALF, blood, and specimen results with thoracic computed tomography (CT) scan essential in making the final conclusion.

It should be also added that SARS-CoV-2, with more than twenty million individuals infected worldwide, still remains unknown in many aspects, which includes the way the virus spreads and cooperates with other pathogens affecting the lungs and different organs such as heart, kidney, liver, and brain. In-fact, metatranscriptomic sequencing performed on BALF obtained from COVID-19 affected patients exposed a significant presence of bacteria highly similar to those found in patients affected by non-COVID-19 pneumonia. In addition to SARS-COV-2 infection, it was seen to contribute to the overall complications and worsening condition of patients with pre-existing comorbidities such as diabetes, cardiovascular disease, cancers, and kidney deficit, often leading to death [6], [7], [8], [9].

In this study, we prepared a comprehensive evaluation of two deceased patients; first patient (negative to swab-RT-PCR) admitted to 118 Pre-Hospital and Emergency Department of SG Moscati Hospital of Taranto City in Southern Italy, which is one of the designated hospitals assigned by Italian Government for patients severely or critically ill with COVID-19. The second patient was a confirmed COVID-19 (positive to swab-RT-PCR) admitted to Emergency Department and then moved to ICU Department of Civic Hospital of Cantu' City in Northern Italy. We aimed to compare these two patients, COVID-19 and COVID-like, both presenting similar clinical features and symptomatology with tight superimposable laboratory and analysis findings, including arterial blood gas (ABG) results and cell blood count (CBC).

\section{Case 1}

The first case was a 51-year-old man, Caucasian admitted to 118 Emergency Hospital of SG Moscati Hospital in Taranto City, transported with "yellow code," in April 24, 2020. The symptoms were dyspnea, tachypnea accompanied by fever, and general malaise that commenced between $19^{\text {th }}$ and $20^{\text {th }}$ of April. At that time, the family Doctor prescribed steroids (betamethasone) and anti-histamines. The situation dramatically declined during the following days to the point had to be admitted into 118 Emergency Department (triage) in the early evening (20:00 pm), where he started receiving the adopted protocol of suspected COVID-19 composed of methylprednisolone, ceftriaxone, enoxaparin sodium, azithromycin, and oxygen therapy.

Of note, the $A B G$ analysis ( $A B G$ ) assessed the presence of $\mathrm{pH}$ alkalosis, with hypoxia and hypocapnia. The $\mathrm{CBC}$ revealed a clinical characterized of neutrophilia and lymphopenia associated with high levels of inflammatory markers such as C-reactive protein (CRP), interleukin 6, D-dimer, and troponin with a low level of e-GFR and vitamin D. The nasopharyngeal swab analyzed by RT-PCR was negative and the CT scan showed the ground-glass opacity (Table 1).

At the beginning, the patient responded positively to the therapy and doctors assessed amelioration, the patient was aware, responsive, and attentive; the breathing was also better and, the fever sloped as well. The revised trauma scores were optimal confirmed by the Cincinnati Prehospital Stroke Scale that was normal.

However, around 23:10 same evening, unexpectedly the patient's condition suddenly worsened while he was moving to the Imaging Department to receive a CT scan (Figures 1a and b). Within a short time, the patient started showing symptoms of severe respiratory distress with hypotension, tachypnea, and hypoxemia and almost immediately entered into an irreversible comatose state. The electrocardiogram (ECG) performed at that time showed a sign of tachycardia with right axial deviation suggestive of ongoing respiratory distress and associated with an ongoing myocardial infarction (QRS-T complex) (Figure 2).

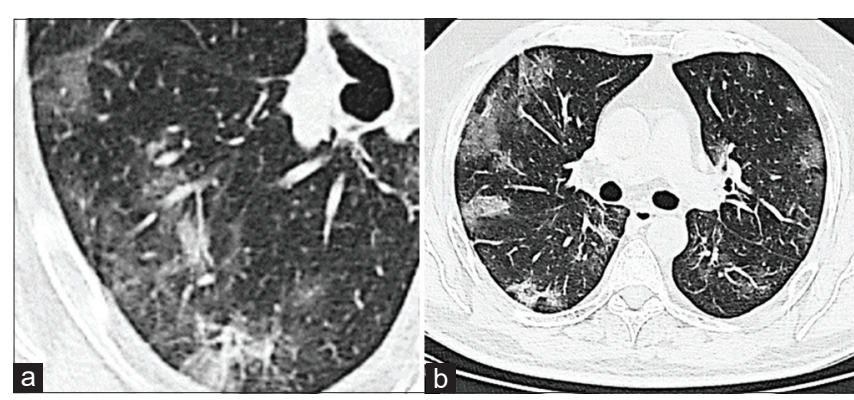

Figure 1: ( $a$ and $b)$ Computed tomography scan of a coronavirus from a patient in SG Moscati showing ground-glass lesions in the lungs bilaterally soon before his situation worsened

The patients died soon after, the death was confirmed by the medical coroner at 00:10 AM of April 25, 2020.

\section{Autopsy examination and findings in case 1}

Autopsy was performed in accordance with Italian NIH protocol and instruction. The whole procedure was completed in a thorough airborne infection isolation autopsy room with the use of appropriate protective tools and shields [10].

The trunk and abdomen were covered by hyperemic patches in a layer-like distribution. The heart presented few abnormal features described as follow: 


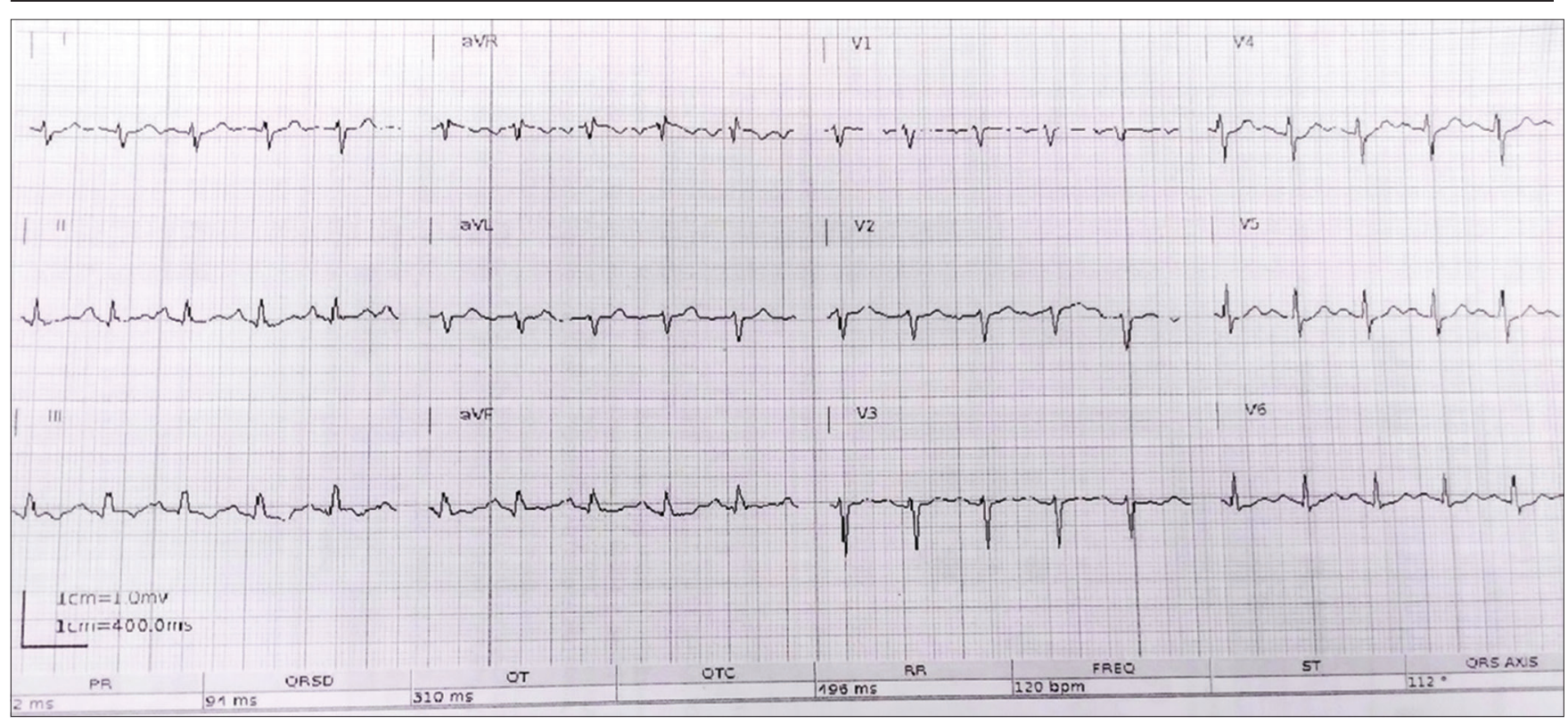

Figure 2: The electrocardiogram (ECG) performed at the time of the admission showed tachycardia with a right axis deviation and evident problem in the ventricular repolarization mechanism. The precordial V1-V6 was suggestive of myocardial infarction in process. In general, the ECG has confirmed a key tool in prognostic value in coronavirus disease (COVID)-19 patients showing a variety of underlying cardiovascular conditions, such as hypertension, offering a particularly attractive methodology current COVID-19 pandemic [9]

Table 1: The vital signs at the time of the entrance of patient $n$. 1 (COVID-like)

\begin{tabular}{|c|c|c|c|}
\hline Patient 1 male 51 years & Vital signs - normal ranges & CT scan & RT-PCR swab \\
\hline Arterial pulse & $140 / 100$ & Ground glass & negative \\
\hline Body temp. & $37.5^{\circ} \mathrm{C}$ & & \\
\hline Heart rate & $120 /$ min & & \\
\hline Respiratory rate & 22. $\min$ & & \\
\hline $\mathrm{O}_{2}$ sat. & $85 \%$ & & \\
\hline Glucose & $119 \mathrm{mg} / \mathrm{dL}(74-100)$ & & \\
\hline \multirow[t]{3}{*}{$A B G$} & pH $7.493(7.35-7.45)$ & & \\
\hline & $\mathrm{PO}_{2} 52.4(75-100)$ & & \\
\hline & $\mathrm{PCO}_{2} 25.3(35-45)$ & & \\
\hline eGFR & $83,73 \mathrm{ml} / \mathrm{min}(>90)$ & & \\
\hline Total bilirubin & $2.20 \mathrm{mg} / \mathrm{dl}(0.50)$ & & \\
\hline Direct bilirubin & $040 \mathrm{mg} / \mathrm{dl}(0.20)$ & & \\
\hline Indirect bilirubin & $180 \mathrm{mg} / \mathrm{dl}(0.30)$ & & \\
\hline Fibrinogen & $586 \mathrm{mg} / \mathrm{dl}(200-400)$ & & \\
\hline Troponin & $54.400 \mathrm{ng} / \mathrm{L}(<14)$ & & \\
\hline CRP & $28.2 \mathrm{mg} / \mathrm{L}(<3.5)$ & & \\
\hline D-dimer & $13.30 \mathrm{mg} / \mathrm{L}(<0.5)$ & & \\
\hline $25 \mathrm{OH}$-vitamin D & $23.1 \mathrm{ng} / \mathrm{ml}(>45)$ & & \\
\hline IL-6 & $222.3 \mathrm{pg} / \mathrm{ml}(<7)$ & & \\
\hline Total WBC & $15.21 \mathrm{10}^{3} / \mathrm{mm}^{3}(3.59-10)$ & & \\
\hline Neutrophilia & $80.7 \%(35-75)$ & & \\
\hline Lymphopenia & $114 \%(20-55)$ & & \\
\hline
\end{tabular}

Globular shape and manifestly enlarged; abnormal condition of the oval foramen was seen as well; right atrial was enlarged and presented a concentrically hypertrophy of ipsilateral ventricle (thickness $1.2 \mathrm{~cm}$ ); it was also seen the stenosis of pulmonary valve consequent of partial fusion of either right or left cuspid valves.

The lungs were found pleura-parietal and pleura-diaphragmatic adherent synechiae with modest pericardial serum effusion of about $100 \mathrm{cc}$. Upon macroscopic evaluation, the lungs were heavily congested and edematous. Bilaterally, the lower lobes showed topographies with dark-red colors consistently with hemorrhagic events, upper lobes were pink with atelectasis facets. Features were suggestive of an exudative and diffuse alveolar damage due to massive thromboembolism found in the right pulmonary artery with multiple occlusions of pulmonary arteries bilaterally mainly seen in the lower lobes. Thus, clots were located in the larger branches as well as in the periphery which means the clots were either thrombotic or embolic. It was confirmed the presence of aortic atherosclerosis.

\section{Case 2}

The second case was an 86-year-old man, Caucasian admitted to 118 Emergency Unit of Civic Cantu' Hospital in Cantu' City Northern Italy. The patient was accepted in "yellow code," in March 17, 2020, at 15:37. The assessed symptoms were dyspnea $\left(\mathrm{PaO}_{2}\right.$ $87 \%$ ) with breathing difficulties, tachycardia, together with fever, asthenia, anosmia, and loss of appetite accompanied by diarrhea, the symptoms commenced March $10^{\text {th }}$. The patient was sent to receive CT scan and blood test analysis. The CT scan revealed multiple small patches of ground-glass opacities, while the blood count results were considered all within the normality ranges. The patient had a story of ischemic attack and benign prostate hyperplasia, he was under anti-coagulants and anti-prostatic medications, and he started receiving antibiotics ceftriaxone. The patient was reacting well to the first line of therapy and doctors assessed a slight amelioration, the patient was aware and attentive, the breathing was as well. However, at 18:37, the patient started worsening and was considered critical $\left(\mathrm{PaO}_{2}\right.$ $67 \%$ ); thus, he received the CPAP $\mathrm{O}_{2}$ mask. Later that night, condition decline further and CPAP was removed and an MV at $50 \%$ was instead positioned to the patient. The day after, March $18^{\text {th }}$, at 9:00 in the morning patient was considered non-responsive. Around 02:19 in the 
early morning of March $21^{\text {st }}$ the patient was eventually assessed deceased.

\section{The vital signs at the time of the entrance were as follow}

The patient was continuously monitored during the time of his hospitalization. The first total

Table 2: Results obtained from patient n.2 (COVID-19)

\begin{tabular}{|c|c|c|c|}
\hline Patient 2 male 86 years & Vital signs - normal range & CT scan & RT-PCR swab \\
\hline \multicolumn{4}{|l|}{ First check } \\
\hline Arterial pulse & $160 / 90$ & Ground glass & positive \\
\hline Body temp. & $36.3^{\circ} \mathrm{C}$ & & \\
\hline Heart rate & $120 /$ min & & \\
\hline Respiratory rate & $22 / \min$ & & \\
\hline $\mathrm{O} 2$ sat. & 91 & & \\
\hline Glucose & $115 \mathrm{mg} / \mathrm{dL}(74-100)$ & & \\
\hline \multirow[t]{3}{*}{$A B G$} & pH 7.568 (7.35-7.45) & & \\
\hline & $\mathrm{PO}_{2} 30.5(75-100)$ & & \\
\hline & $\mathrm{PCO}_{2} 18.1(35-45)$ & & \\
\hline eGFR & $46.20 \mathrm{ml} / \mathrm{min}(>90)$ & & \\
\hline Creatine & $1.15 \mathrm{mg} / \mathrm{dl}(0.6-1.3)$ & & \\
\hline Chlorine & $99 \mathrm{mEq} / \mathrm{L}$ & & \\
\hline Second check & Vital signs - normal range & & \\
\hline Glucose & $180 \mathrm{mg} / \mathrm{DI}(74-100)$ & & \\
\hline eGFR & $37.1 \mathrm{ml} / \mathrm{min}(>90)$ & & \\
\hline Fibrinogen & $602 \mathrm{mg} / \mathrm{dl}(200-400)$ & & \\
\hline Urea & $81 \mathrm{mg} / \mathrm{dl}$ & & \\
\hline Troponin & $6337 \mathrm{ng} / \mathrm{L}(<14)$ & & \\
\hline CRP & $225.7 \mathrm{mg} / \mathrm{L}(<3.5)$ & & \\
\hline Pro-BNP & $18800 \mathrm{pg} / \mathrm{ml}(<450)$ & & \\
\hline D-dimer & $51977 \mathrm{ng} / \mathrm{ml}(<0.5)$ & & \\
\hline p-PT/INR & $1.55(<1.1)$ & & \\
\hline total WBC & $20.1610^{\wedge} 3 / \mathrm{mm}^{3}(3.59-10)$ & & \\
\hline Neutrophilia & $89.9 \%(35-75)$ & & \\
\hline Lymphopenia & $5.3 \%(20-55)$ & & \\
\hline Potassium & $3.20 \mathrm{mEq} / \mathrm{L}(3.5-5.2)$ & & \\
\hline AST & $90 \mathrm{U} / \mathrm{L}(40)$ & & \\
\hline ALT & $57 \mathrm{U} / \mathrm{L}(55)$ & & \\
\hline LDH & 1095 U/I (100-245) & & \\
\hline CPK & 418 UI/L ( & & \\
\hline CK-MB & $11.70 \mathrm{ng} / \mathrm{ml}$ & & \\
\hline
\end{tabular}

CBC performed March 11, 2020, showed only a few abnormal parameters (Table 2).

The $C B C$ and vital signs at the entrance showed a not alarming situation, with an arterial pulse of $160 / 90$, no fever $36.3^{\circ} \mathrm{C}$, the heart rate was acceptable with $120 \mathrm{~b} / \mathrm{min}$ and respiratory rate $22 / \mathrm{min}$. The patient was considered alert and responsive to stimuli; the revised trauma scores were normal; the Cincinnati Prehospital Stroke Scale was also normal (Table 2 for other levels and signs). However, the patients were saturating very low with an $\mathrm{O}_{2}$ level of $91 \%(A A)$, the $A B G$ analysis confirmed a critical condition with typical signs of $\mathrm{pH}$ alkalosis 7.568, hypoxia $\mathrm{PO}_{2} 30.5$, and hypocapnia $\mathrm{PCO}_{2} 18.1$. The ECG performed at the time of admission confirmed a sinus tachycardia with typical signs of an ongoing $\mathrm{Ml}$ prevalently seen in precordial V1-6 (QRS-T complex) (Figure 3).

The second total CBC performed March 17, 2020 , showed a completely different clinical scenario all metabolic, chemistry, and inflammatory parameters were suggestive of a fast worsening condition (Table 2).

\section{Thoracic CT scan}

The CT scan findings of COVID-19 are those of atypical pneumonia or organizing pneumonia. Pulmonary hilar were congested and enlarged in appearance, it was seen a diffuse reticular-nodular with a parenchymal consolidation characterized by bilateral ground-glass opacities evidently the expression of active inflammation (Figure $4 a$ and b).

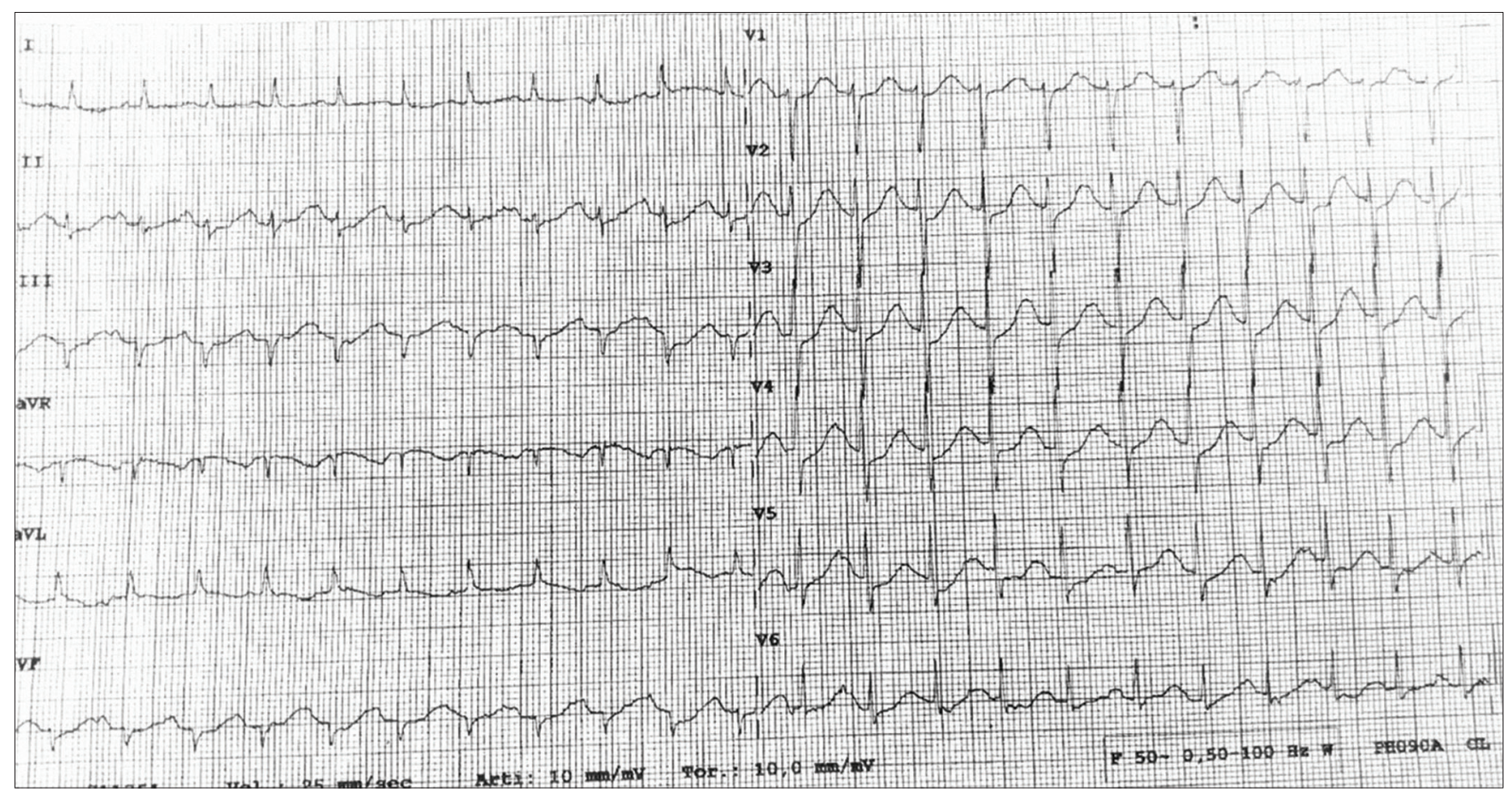

Figure 3: The electrocardiogram performed at the time of the admission showed sinus tachycardia with right atrial functionality. The precordial V1-V6 was indicative of myocardial infarction in process with ongoing angina. The patient died a few hours after for a massive severe cardiorespiratory collapse 


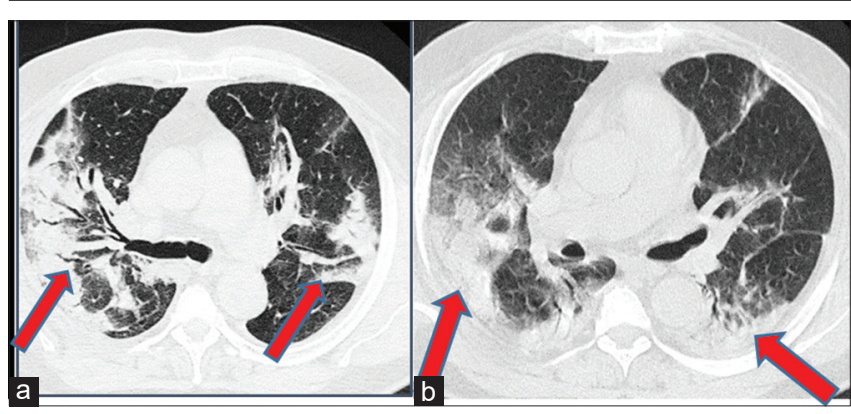

Figure 4: ( $a$ and b) Computed tomography scan obtained on illness day 1 shows a mixed pattern with an evident ground glass extent and atelectasis (red arrows) bilateral. The overall consolidation suggests the presence of organizing interstitial pneumonia

\section{Discussion}

Due to the rapid spread and the increasing number of COVID-19 infected patients, there is the need of more accurate evaluation of the virus to better control the sources of infection helping the population in preventing disease progression and spreading. Since December 2019, there have been considerable challenges especially in regarding the use of nucleic acid test (the nasal-pharyngeal swab by RT-PCR procedure), the CT scan, and the clinical physiognomies in trying to produce a standard orientation toward a more definitive diagnose of COVID-19 disease [11].

Nevertheless, at present, the diagnosis cornerstone remains in COVID-19 early-onset the positivity of RT-PCR and (only as secondary) the CT scan with the lung "ground glass" images and ABG analysis. This procedure would tend to exclude all those types of patients that we have titled COVID-like, which exhibit analogous symptoms and clinical picture of COVID-19 disease. The consequence of this diagnostic discrimination would eventually pose two fundamental and practical problems; first, the denial of having contracted the virus and thus being a potential contagious threat and second, the renunciation to consider the crucial results from the $A B G$ and $C T$ scan as predictive of more general worsening of the clinical condition due to virus infection.

The COVID-19 and COVID-like patients share many features, they manifest low-intensity symptoms that usually aggravate shortly before the admission to the hospital, commencing with unnoticeable episodes of increasing respiratory difficulties. Furthermore, the COVID-19 and COVID-like patients show also similar clinical and laboratory patterns, low fever, dry cough, and medium intensity headache; the $A B G$ analysis shows an alkalotic $\mathrm{pH}$, with low $\mathrm{PaO}_{2}$ and low $\mathrm{PaCO}_{2}$, low lymphocytes, high neutrophils, high fibrinogen, high troponin, high CRP, high D-dimer, low eGFR, and high ESR. Apparently, both types of patients seem to respond well to the preliminary therapy approach, and both types of patients reveal a similar sudden and unexpected deteriorating process that may end-up to severe hypoxia, hypocapnia, MOF, and then death.
In these short two case reports, our main aim was to highlight the similarities between COVID-19 and COVID-like deceased. COVID-like and COVID-19 share many common pattern of the infection much more than COVID-like would share with other pulmonary disease. Alarm has risen, especially after considering the deceptive good health of COVID-like individuals, which may become an exceptional contagious source. Important, the autopsy findings and post-mortem laboratory results performed on both COVID-19 and COVID-like deceased patients were all suggestive of a death consequent of an acute cardio-respiratory decay characterized by massive pulmonary thromboembolism accompanied by a considerable pulmonary hemorrhage as finally reported by few prominent published studies [13], [14], [15], [16].

\section{Conclusion}

While we are still learning and close to a second COVID-19 pandemic wave, there is still a lot that remains to be clarified about SARS-CoV-2. These short descriptive two case reports, which is just a part of wider and more extensive study yet to be published, are an attempt of comparing COVID-19 and COVID-like disease as we strongly believe that it would be of great importance to identify patients at very high risk of mortality as a result of an overlapping of risk factors that were separately reported in patients who died from COVID-19 or COVID-like. Subjects aged $\geq 45-90$ years and suffering from pre-existing comorbidities such as diabetes, cardiovascular diseases, or kidney disorders are likely to be the elective target of prevention and diagnostic activities. This is the reason why an improved investigation is mandatory, in which RT-PCR and CT scan procedures are completed by data from more detailed laboratory analysis, ABG analysis, BALF, and a deeper clinical assessment.

\section{Consensus}

This brief report received an official written consensus from the family of the deceased patients.

\section{References}

1. Santacroce L, Charitos IA, Del Prete R. COVID-19 in Italy: An overview from the first case to date. Electron $\mathrm{J}$ Gen Med. 
2020;17(6):em235. https://doi.org/10.29333/ejgm/7926

2. Ballini A, Dipalma G, Isacco CG, Boccellino M, Di Domenico M, et al. Oral microbiota and immune system crosstalk: $A$ translational research. Biology (Basel). 2020;9(6):131.

3. Pham HV, Isacco CG, Nguyen CD, Le SH, Tran DK, Nguyen QV, et al. Rapid and sensitive diagnostic procedure for multiple detection of pandemic coronaviridae family members SARSCoV-2, SARS-CoV, MERS-CoV and HCoV: A translational research and cooperation between the Phan Chau Trinh university in Vietnam and university of Bari "Aldo Moro" in Italy. Eur Rev Med Pharmacol Sci. 2020;24(12):7173-91. https://doi. org/10.7717/peerj.9725/table-3

PMid:32633414

4. Charitos IA, Ballini A, Bottalico L, Cantore S, Passarelli PC, Inchingolo $\mathrm{F}$, et al. Special features of SARS-CoV-2 in daily practice. World J Clin Cases. 2020;8(18):3920-33. https://doi. org/10.12998/wjcc.v8.i18.3920

PMid:33024749

5. Geng YJ, Wei ZY, Qian HY, Huang J, Lodato R, Castriotta RJ. Pathophysiological characteristics and therapeutic approaches for pulmonary injury and cardiovascular complications of coronavirus disease 2019. Cardiovasc Pathol. 2020;47:107228. https://doi.org/10.1016/j.carpath.2020.107228

PMid:32375085

6. Shen Z, Xiao Y, Kang L, Ma W, Shi L, Zhang L, et al. Genomic diversity of severe acute respiratory syndrome-coronavirus 2 in patients with coronavirus disease 2019. Clin Infect Dis. 2020;71(15):713-20.

PMid:32129843

7. Du Y, Tu L, Zhu P, Mu M, Wang R, Yang $P$, et al. Clinical features of 85 fatal cases of COVID-19 from Wuhan. A retrospective observational study. Am J Respir Crit Care Med. 2020;201(11):1372-9. https://doi.org/10.1164/ rccm.202003-0543oc

PMid:32242738

8. Vermesan D, Vermesan $\mathrm{H}$, Dragulescu SI, Bera I, Di Giovanni A, Sabatini R, et al. Secondary pathologic fractures in osteosarcoma: Prognosis and evolution. Eur Rev Med Pharmacol Sci. 2009;13(2):71-6.

PMid:19499840
9. Guan WJ, Ni ZY, Hu Y, Liang WH, Ou CQ, He JX, et al. Clinical characteristics of coronavirus disease 2019 in China. N Engl J Med. 2020;382:1708-20.

10. McCullough SA, Goyal P, Krishnan U, Choi JJ, Safford MM, Okin PM. Electrocardiographic findings in coronavirus disease-19: Insights on mortality and underlying myocardial processes. J Card Fail. 2020;26(7):626-32. https://doi. org/10.1016/j.cardfail.2020.06.005

11. Carsana L, Sonzogni A, Nasr A, Rossi RS, Pellegrinelli A, Zerbi P, et al. Pulmonary post-mortem findings in a series of COVID-19 cases from Northern Italy: A two-centre descriptive study. Lancet Infect Dis. 2020;20:1135-40. https://doi.org/10.1016/ s1473-3099(20)30434-5

12. Santacroce $L$, Charitos IA, Ballini A, Inchingolo F, Luperto $P$, De Nitto $E$, et al. The human respiratory system and its microbiome at a glimpse. Biology (Basel). 2020;9(10):318. https://doi. org/10.3390/biology9100318.

PMid:33019595

13. Tahamtan A, Ardebili A. Real-time RT-PCR in COVID-19 detection: Issues affecting the results. Expert Rev Mol Diagn. 2020;20(5):453-4. https://doi.org/10.1080/14737159.2020.17 57437 PMid:32297805

14. Di Serio F, Lovero R, D'Agostino D, Nisi L, Miragliotta G, Contino R, et al. Evaluation of procalcitonin, Vitamin $\mathrm{D}$ and C-reactive protein levels in septic patients with positive emocoltures. Our preliminary experience. Acta Med Mediterr 2016;32:1911-4

15. Lodigiani $C$, lapichino $G$, Larenzo L, Cecconi M, Ferrazzi $P$, Sebastian T, et al, Humanitas COVID-19 Task Force. Venous and arterial thromboembolic complications in COVID-19 patients admitted to an academic hospital in Milan, Italy. Thromb Res. 2020;191:9-14. https://doi.org/10.1016/j.jvsv.2020.08.013 PMid:32353746

16. Al-Samkary $\mathrm{H}$, Karp-Leaf RS, Dzick WH, Carlson JC, Fogerty $\mathrm{AE}$, Waheed $\mathrm{A}$, et al. COVID-19 and coagulation: Bleeding and thrombotic manifestations of SARS-CoV-2 infection. Blood. 2020;136(4):489-500. https://doi.org/10.1182/ blood.2020006520

PMid:32492712 